\title{
A Dyadic Perspective on Coping and its Effects on Relationship Quality and Psychological Distress in Couples Living with Chronic Pain: A Longitudinal Study
}

Manasi M Mittinty, $\mathrm{MD}, \mathrm{PhD}^{1-2}$, Sarah Kindt, $\mathrm{PhD}^{2}$, Murthy $\mathrm{N}$ Mittinty, $\mathrm{PhD}^{3}$, Sonia Bernardes, $\mathrm{PhD}^{4}$, Annmarie Cano, $\mathrm{PhD}^{5}$, Lesley Verhofstadt, $\mathrm{PhD}^{2}$, Liesbet Goubert, $\mathrm{PhD}^{2}$

1. Pain Management Research Institute, The University of Sydney, Australia

2. Department of Experimental-Clinical and Health Psychology, Ghent University, Belgium

3. School of Public Health, The University of Adelaide, Australia

4. Department of Social and Organisational Psychology, Instituto Universitario de Lisboa, Lisbon, Portugal

5. Department of Psychology, Wayne State University, Detroit, MI, USA

Correspondence: Manasi M Mittinty

Lecturer, Pain Management Research Institute, The University of Sydney, Australia

E: manasi.mittinty@sydney.edu.au

Type of Article: Original Research

Total number of pages: 46

Total number of Figure: 1

Total number of Table: 3

Funding sources:

This research was supported by the Fund for Scientific Research-Flanders (FWO), grant number G.0235.13N. MM Mittinty is funded by the Endeavour Post-doctoral research fellowship, Department of Education and Training, Australian Government, Australia.

Conflict of interest

The authors have no conflicts of interest to disclose. 


\begin{abstract}
Objective. Dyadic coping is a process of coping within couples that is intended not only to support the patient with chronic pain but also to maintain equilibrium in the relationship. This study aims to investigate the effect of patient-perceived and spouse-reported dyadic coping on both patient and their partner's relationship quality and anxiety, stress, depression, over time. Methods. 139 couples, with one partner experiencing chronic pain, participated in this study. Spanning three measurements over six months, couples reported on their anxiety, stress, depression, relationship quality and dyadic coping. Results. Patient-perceived supportive dyadic coping was positively associated with both partners' relationship quality but was negatively associated with spouses' stress, over time. Patient-perceived negative dyadic coping was negatively associated with both partners' relationship quality and positively associated with patients' depression and spouses' depression and stress, over time. Spouse-reported supportive dyadic coping showed a positive association with their own relationship quality and a negative association with spouses' depression at baseline and, patients' depression at 3 months follow up. Spouse-reported negative dyadic coping was negatively associated with their relationship quality at baseline and positively associated with their partner's anxiety and stress at 6 months and 3 months follow up, respectively. Similar inference was observed from the findings of growth curve model. Conclusions. As compared to spouse report, patient perception of dyadic coping is a better predictor of both partners' relationship quality, and psychological outcomes, over time. Both partners may benefit from early psychosocial intervention for improving their dyadic coping, and also their relationship quality and psychological outcomes.
\end{abstract}

\title{
Key Words.
}

Chronic pain couples, Dyadic coping, relationship quality, anxiety, stress, depression, psychological distress 


\section{Introduction}

Chronic pain is a critical health condition which has deleterious effects on patients' functional, psychological and social wellbeing [1-3]. Approximately one third of the global population suffers from chronic pain [4]. Despite several advancements in understanding pain, its optimal management still eludes patients' and health care providers alike. There has been considerable conjecture on the role social environment plays on patients' adaption and recovery. Given that partners are often an important source of support [5], understanding the role of partners' coping efforts and support provision remains an important goal of current research. Research on partner support has demonstrated that volitionally provided spousal support (autonomous support provided by spouse out of commitment and affection) is perceived as more helpful by the patients than support provided under pressure (support provided out of guilt or criticism), and is associated with improved wellbeing, reduced stress [6], and better physical and psychological functioning [7-9]. Although timing and motivation behind partner support has been investigated, very little is known about the bi-directional nature of dyadic coping which comprises of appraisal and response to pain from both partners [10].

According to Bodenmann's Systemic Transactional Model (STM) [11], dyadic coping that transpires between couples is a transactional process wherein both partners employ strategies intended to not only help the ill partner but also to maintain equilibrium in their relationship $[11,12]$. The present literature on dyadic coping shows contradictory findings, with some studies suggesting beneficial roles of partner provided coping, wherein patients experience lower pain [13] and higher resilience [14-16], to some studies demonstrating that partners' responses and coping efforts can have negative effects on patients' experience of pain [17] and can cause more anxiety and depressive symptoms [18-20]. Although none of these studies have identified direct associations between dyadic coping and patient-related outcomes they do 
demonstrate that the partner's coping efforts are not always deemed beneficial [21] and may underlie psychological distress [22,23] and marital discord [20] between the couple. An alternate possibility is that dyadic coping could more directly be associated with relationship outcomes and indirectly associated with individual outcomes. Moreover, existing research puts greater emphasis on the impact of dyadic coping on patient-related outcomes while very little is known about how dyadic coping affects their partner's wellbeing and relationship quality. Traditionally, coping with chronic pain requires significant adjustment from both the patient and their partner. Partners who often become the primary caregiver and source of emotional support for the patients while also dealing with their own emotions of witnessing their loved one suffer make significant adjustments and changes to their daily living and also long-term life plans. Pain becomes a shared stressor between the couple who confer shared meaning to the experience and employ mutual coping strategies [24]. This dyadic conceptualization of coping with pain recognizes the interdependence between intimate relationships and opens a new understanding of how coping with pain can affect their relationship and wellbeing.

Therefore, in order to fully capture the impact of dyadic coping, it is necessary to examine the process from the perspective of both members of the dyad. Commonly, three types of associations are observed in dyadic data, namely interrelation associations (associations observed among multiple variables e.g. relationship quality and dyadic coping), temporal associations (associations between data collected at multiple times) and interpersonal associations (associations between observations coming from members of a social unit such as couple, family).

Furthermore, most research to date on spousal support has been cross-sectional, with a call for prospective studies [5]. To address these gaps, the present study applies the STM model of dyadic coping and examines both patient and their partners' perspective on dyadic coping, using a longitudinal study design with a follow-up period of 6 months. The specific aims of the 
study are to examine: (i) the interrelations associations between patient-perceived dyadic coping at baseline and patient and their partner's psychological and relationship outcomes at baseline (Model 1), 3 months (Model 2) and 6 months (Model 3) follow up, (ii) the interrelations associations between spouse-reported dyadic coping at baseline and patient and spouses' psychological and relationship outcomes at baseline (Model 1), 3 months (Model 2) and 6 months (Model 3) follow up and (iii) the temporal and interpersonal associations between, (a) supportive and negative dyadic coping and (b) psychological outcomes and (c) relationship outcomes as reported by both patient and their spouse.

\section{Methods}

\section{Design, Study Setting and Participants}

The present study was conducted as a part of the "Helping Motivation Diary and Longitudinal Study", by the Gent Health Psychology Lab. Members of the Flemish League for Fibromyalgia Patients and the Flemish Pain League were invited via a letter to participate in this study. To be eligible to participate in the study; (i) patients had to be experiencing chronic pain for at least 3 months, (ii) living together with a partner for more than a year and (iii) both partners had to be proficient in Dutch language. The participating couples were asked to complete the questionnaires at three different time points; baseline (T1), 3 months after T1 (T2), and 6 months T1 (T3). At baseline, data were collected via home visits (1 - 1,5 hour), as it also facilitated collection of informed consents. Completing the questionnaires took approximately 30 to 45 minutes. Follow up data at time T2 and T3 were collected using a secured online survey tool called LimeSurvey. During the baseline data collection, all couples were explained that it was necessary that each partner completes their questionnaires individually so that their responses would not be influenced by the others partner's views. Couples who did not have access to computers or internet, or where not experienced with using computer/internet, were provided with a paper version of the survey. At T1, 39 patients $(27.86 \%)$ and 31 partners 
$(22.14 \%)$ chose to use the paper versions of the questionnaires. All couples received 30 euros on completion of the study at T2 and T3 as token of appreciation. Ethical approval was obtained from the Faculty of Psychology and Educational Sciences, Ghent University. A total of 141 couples participated, of which 97 couples were recruited from the Flemish Pain League (response rate of $83.62 \%$ ) and 44 members were recruited from the Flemish League for Fibromyalgia Patients (response rate of $70.97 \%$ ). Most couples that were contacted by phone and met the inclusion criteria wanted to participate in our study (86.2\% for Flemish Pain League and $69 \%$ for the Flemish League for Fibromyalgia Patients). However, complete couple data were only available for 139 couples at T1, 133 couples at T2 and 127 couples at T3. Lack of interest or time were the main reasons for the dropout. Couples who had missing data either from the patient or partner were excluded from the final analysis. All couples (patient and partner) completed the baseline demographic questionnaire at $\mathrm{T} 1$ and the Dyadic Coping Inventory, the Depression Anxiety Stress Scale, and the Dyadic Adjustment Scale at T1, T2 and T3. In addition, patients also filled out the Graded Chronic Pain Scale (GCPS) at T1, T2 and $\mathrm{T} 3$.

\section{Measures}

\section{Sociodemographic information}

Information about the patients' and their spouses' age, sex, marital status, race, nationality, relationship length (years), education (years), and employment status were collected at T1. Three pain ratings measuring the current, average and worst pain the patients had experienced in the past six months were collected. The pain ratings were measured using the GCPS [25] on a scale of "0" (no pain) to "10" (worst imaginable pain). A score was calculated by averaging all three ratings at each time point. Cronbach's alphas were $.88, .92, .92$ for T1, T2 and T2, respectively.

\section{Pain-specific Dyadic Coping}


Two subscales (supportive and negative dyadic coping) from the Dyadic Coping Inventory (DCI) [26] measuring dyadic coping in couples coping with stress were used [26-27]. The Dyadic Coping Inventory has been tested and validated for use in couples coping with different forms of stress [27, 28, 29]. Reports of dyadic coping from both patient and their spouses were collected at all time points. In this study, the scale was specifically adapted for use in the context of pain by replacing "stress" to "pain" in the instructions and items. The supportive dyadic coping subscale was adapted to capture partners' efforts to assist the patient. Items were adapted as follows to capture the emotion focused efforts, e.g. "I talk to my partner about their pain and help them change their perspective"/ My partner helps me look at my pain differently", or "When my partner is in pain, I offer help/ My partner helps me do things when I am in pain. Similarly, the negative dyadic coping subscale was adapted to capture partners' ambivalent or superficial efforts in assisting the patient, e.g. "I often ignore my partner when he/she is in pain"/When I am in pain, my partner withdraws". Scores were generated by summing each item ( 5 for supportive dyadic coping and 4 for negative dyadic coping) in the scale. In the current study, Cronbach's alpha for supportive dyadic coping reported by patients was .89 for each time point and for supportive dyadic coping reported by partners it was .70 , .68 and .72 for T1, T2 and T3, respectively. Cronbach's alphas for negative dyadic coping were $.79, .86$ and .85 for patients and $.64, .68$ and .71 for partners for T1, T2 and T3, respectively.

\section{Psychological distress}

The Dutch version [30] of the Depression Anxiety Stress Scale (DASS) [31] was used to measure psychological distress, including anxiety (e.g., I experienced trembling in hands), stress (e.g., I found myself getting agitated) and depression (e.g., I found it difficult to work up the initiative do to things). The DASS is a 21 -item scale divided into 3 subscales. Each subscale consists of 7 items rated on a four-point Likert scale from "0" (not at all) to "3" (very much). Scores for each of the three DASS scales were obtained by summing the scores from the 7 
items in the scale, and then multiplying the score by 2 . Higher scores indicate higher levels of anxiety, stress or depressive symptoms. Cronbach's alphas in the current study for patient reports were $.88, .90, .91$ for depression, $.80, .82, .85$ for anxiety and $.88, .91$ and .91 for stress for T1, T2 and T3, respectively. Also, partner-reported psychological distress showed good internal consistency with $.90, .89$ and .92 for depression, $.90, .89$ and .93 for anxiety and .86 , .88 and .88 for stress for T1, T2 and T3, respectively.

\section{Relationship quality}

The Dyadic Adjustment Scale (DAS) [32] was used to assess couple's relationship quality. DAS is a 32-item scale, containing four subscales: dyadic satisfaction, dyadic consensus, dyadic cohesion and dyadic affectional expression. A total score was calculated by adding the scores from all four subscales; a score of 100 is a cutoff point for poor marital functioning. Higher scores reflect higher relationship quality. The DAS has high test-retest reliability, validity and internal consistency (Cronbach's alpha, $\alpha=0.93$ ), which has been confirmed by other studies [6,32-34]. In the current study Cronbach's alpha for patients were $.91, .93$ and .93 for T1, T2 and T3, respectively and for partners it was .90, .92 and .91 for T1, T2 and T3, respectively.

\section{Statistical Analyses}

\section{Actor-Partner Interdependence Model}

An innovative statistical model known as the Actor-Partner Interdependence Model (APIM) was used to examine the interrelations associations. As the data were collected from couples concurrently, the effects of patient-perceived dyadic coping and spouse-reported dyadic coping were examined together by treating data from both members of the dyads as a unit, by using the Actor-Partner Interdependence Model (APIM) [35]. The APIM model combines the interdependence of dyadic relationships with appropriate statistical techniques for measuring and testing it. As a corollary of the interdependence between dyads, the observation of one 
spouses' emotions, behaviors and cognitions affects the emotion, behavior and cognition of the other spouse. This approach allows measuring both individual and dyadic effects also known as the "actor" and "partner" effect, respectively. The actor effect measures the association between an individual's outcome variable score and their predictor variable score. Similarly, the partner effect measures the association between the individual's outcome variable score on their spouses' predictor variable score [36]. Thus, APIM allows to measure interdependence within interpersonal relationships and disentangle an individual effect from a partner effect [35]. To avoid confusion, we have purposefully used the term "spouse" when referring to a patient's partner, and "partner" when referring to the APIM model.

- insert Figure 1 about here -

As seen in Figure 1, there are two actor paths ( $a$ and $b$ ) which represent the influence of perceived (supportive and negative) dyadic coping and reported (supportive and negative) dyadic coping on patient and spouses' relationship quality and psychological distress, respectively. There are also two partner-paths (c and d) which represent the effects of perceived (supportive and negative) dyadic coping on spouses' outcomes (path c) and the effect of reported (supportive and negative) dyadic coping on patients' outcomes (path d).

Following Griffin \& Gonzalez (2001), the seemingly unrelated regression (SUR) in STATA was used to fit APIM to the data. SUR was chosen in view of its ability to handle correlated errors of the regressions between patient and spouse responses. All outcome regressions were adjusted for patients' sex, pain intensity, and relationship duration. Three models were tested, Model 1 tested the association between patient-perceived and spouse-reported dyadic coping at baseline on patient and spouse related outcomes at baseline, Model 2 tested the association between patient-perceived and spouse-reported dyadic coping at baseline on patient and spouse related outcomes at 3 months follow up and Model 3 tested the association between patient- 
perceived and spouse-reported dyadic coping at baseline on both the patient and the spouse related outcomes at 6 months follow up. In Model 2 and Model 3, each outcome at baseline was controlled for when examining its longitudinal associations at 3 and 6 months respectively.

\section{Dyadic Growth Curve Model}

Traditional statistical methods such as linear regression are developed using independent and identical data. For analysing information on dyad use of these traditional methods is inappropriate as the data are correlated (interpersonal associations). To this extent one can either use Structural equation models or growth curve models (e.g. Generalised estimating equations). In addition to dyads if one also has repeated measures (temporal associations), as we do in our data, one can use intensive longitudinal methods such as dyadic growth curve models. Hence, dyadic growth curve modelling was used to examine the interpersonal and temporal associations. In this modelling the data of repeated measures is nested within a person and each person is nested within a dyad [37]. For example spouse information is nested within time and patient information is nested within time and then both the spouse and patient are nested within a couple id. Such nesting is similar to a multilevel model (MLM), where the first level is individual, second level is time and the third level being the couple. Here, the couple are assumed to be independently sampled. MLM is a method for analysing hierarchically nested data structures such as dyads. Non-independence of the members of the dyad and across the repeated occasions are modelled explicitly. This modelling now allows us to answer questions such as

- Is the supportive dyadic coping associated with relationship satisfaction?

$\circ$ What is the extent of association for the spouse and for the patient (fixed effects)? 
All analysis were conducted in R using the nonlinear mixed effects models (nlme) package. The basic multilevel equation can be expressed as

$$
Y_{i j k}=m_{k}+a_{i k}+b_{j k}+g_{i j k}
$$

Where $Y_{i j k}$ is the outcome (e.g. relationship score) for person $i$ rating person $j$ in time $k$. In this equation $m_{k}$ is the group mean, $a_{i k}$ is individual $i$ 's actor effect, $b_{j k}$ is individual $j$ 's partner effect, and $g_{i j k}$ is the relationship or actor-partner interaction effect. In our model we assumed that $m_{k}$ to be zero indicating that the model is passing through origin. The terms $a, b, g$, are random variables and each has a variance $\left(\sigma_{m}^{2}, \sigma_{a}^{2}, \sigma_{b}^{2}, \sigma_{g}^{2}\right)$. The multilevel model also specifies correlations such as spouse effect correlated with the patient effect. Using these models we have now conducted the analysis for testing the associations between psychological (stress, depression) and relationship outcomes. R code used in this computation can be provided upon request.

\section{Results}

\section{Participant characteristics}

Only data from couples who had complete data at all three time points $(n=139)$ were used for the main results. Of the final sample, $82 \%(n=114)$ of patients were female and the $81 \%$ of spouses were male. The mean age of patients and spouses was $52.4(\mathrm{SD}=11.7)$ and 53.6 years $(\mathrm{SD}=12.02)$, respectively. All couples identified as Caucasian. All but two couples reported being in a different-sex relationship. The majority of the participants were married or cohabitating $(82 \%)$, with mean length of relationship in years 25.2 years $(\mathrm{SD}=14.96)$. More than one-third of the couples reported an education beyond the age of 18 years $(37 \%$ for patients; $34.3 \%$ for spouse). Sixty percent of the patients were unemployed while the majority of spouses were in full time employment $(65 \%)$. Most patients reported pain in more than one 
location, with back pain (90\%), neck pain (75\%) and lower extremities (62\%) reported most frequently. The mean pain intensity for patients was $6.90(\mathrm{SD}=1.41)$.

\section{Actor Effects of patient-perceived dyadic coping on patients' relationship quality and}

\section{psychological distress}

Patient-perceived supportive dyadic coping showed strong positive associations with their own relationship quality at baseline $(\beta=0.25, \mathrm{p}=0.01), 3$ months $(\beta=3.05, \mathrm{p}=0.01)$ and 6 months ( $\beta=3.66, p=0.01)$ follow up. This finding suggests that patients who reported receiving more supportive dyadic coping from their spouse were more likely to experience higher relationship quality, over time. However, no significant association was observed between patientperceived supportive dyadic coping and their psychological distress at any time point (see Table 1A).

Patient-perceived negative dyadic coping showed strong negative associations with their own relationship quality at baseline $(\beta=-3.27, \mathrm{p}=0.01)$, at 3 months $(\beta=-4.93, \mathrm{p}=.01)$ and 6 months $(\beta=-4.34, \mathrm{p}=.01)$ follow up. The direction of these associations suggests that patients who reported that they received more negative dyadic coping at baseline from their spouse progressively reported worsening in their relationship quality, over time. In addition, patientperceived negative dyadic coping also showed a significant positive association with depression at baseline $(\beta=.59, \mathrm{p}=.01)$ and 6 months follow up $(\beta=1.24, \mathrm{p}=.01)$, suggesting that patients may experience more depressive symptoms up to 6 months if they perceive that their spouse is providing them with more negative dyadic coping. No cross-sectional or prospective effect of patient-perceived negative dyadic coping was observed on patients' anxiety and stress (refer Table 1A). 


\section{Partner Effects of Patient-perceived dyadic coping on spouses' relationship quality and}

\section{psychological distress}

Patient-perceived supportive dyadic coping showed strong positive associations with their spouses' relationship quality at baseline $(\beta=1.32, \mathrm{p}=0.01)$, at 3 months $(\beta=1.51, \mathrm{p}=0.01)$ and 6 months $(\beta=2.27, p=0.01)$ follow up. Patient-perceived supportive dyadic coping was negatively associated with spouses' stress at 3 months $(\beta=-0.57, p=0.04)$ and 6 months $(\beta=-$ 0.95, $\mathrm{p}=0.01$ ) follow up. This suggests that spouses of patients who report receiving more supportive dyadic coping are more likely to report good relationship quality and lower stress, over time. However, no statistically significant association was observed between patientperceived supportive dyadic coping and spouses' report of anxiety and depression (refer Table 1B).

Patient-perceived negative dyadic coping demonstrated strong negative associations with spouses' relationship quality $(\beta=-1.97, \mathrm{p}=.01)$ at baseline, at 3 months $(\beta=-1.97, \mathrm{p}=.01)$ and 6 months $(\beta=-2.77, p=.01)$ follow up. The direction of these associations between perceived negative dyadic coping and spouses' relationship quality remained negative suggesting that spouses of patients who perceive receiving higher negative dyadic coping at baseline progressively report worsening in their relationship quality, over time. Patient-perceived negative dyadic coping also showed a strong positive association with spouses' depression $(\beta=.63, p=.01)$ at baseline and stress $(B=0.85, p=.02)$ at 6 months follow up (refer Table $1 B)$. This suggests that spouses of patients who report receiving more negative dyadic coping are more likely to experience at least one psychological distress outcome, over time.

Actor Effects of spouse-reported dyadic coping on spouses' relationship quality and psychological distress

Spouse-reported supportive dyadic coping showed a strong positive association with their own relationship quality $(\beta=1.51, p=.01)$ and a negative association with depression $(\beta=-0.59$, 
$\mathrm{p}=.01)$ at baseline. However, this effect was lost over time at 3 months and 6 months follow up. This finding suggests that spouses who reported providing more supportive dyadic coping were more likely to experience better relationship quality and lower depression at baseline, but this effect was transient and was lost over time (refer Table 2A).

Spouse-reported negative dyadic coping also showed a strong negative association with their relationship quality $(\beta=-1.38, \mathrm{p}=.01)$ and a positive association with depression $(\beta=.69, \mathrm{p}=.01)$ at baseline. However, this association was not observed at 3 and 6 months follow up. However, spouse-reported negative dyadic coping, assessed at baseline, showed significantly positive associations with anxiety $(\beta=1.18, p=.01)$, and stress $(\beta=1.16, p=.01)$ at 3 months follow up but not at 6 months follow up. This finding suggests that providing more negative dyadic coping not only had an immediate effect on spouses' relationship quality but showed continued impact on at least one indicator of psychological distress until 3 months later (refer Table 2A).

\section{Partner effects of spouse-reported dyadic coping on patients' relationship quality and}

\section{psychological distress}

Spouse-reported supportive dyadic coping showed a negative association with patients' depression at 3 months follow up ( $\beta=-0.93, p=0.03)$, which was lost at 6 months follow up. No statistically significant association between spouses' report of supportive dyadic coping and patients' relationship quality, anxiety and stress were observed. This finding suggests that spouses' own report of providing supportive dyadic coping had no immediate or over time effect on patients' relationship quality and psychological distress, except for the association with depressive symptoms at 3 months follow up (refer, Table 2B).

Spouse-reported negative dyadic coping showed positive associations with patients' anxiety at baseline $(\beta=0.72, p=.01)$ and 6 months follow up $(\beta=1.17, p=0.01)$, and stress at 6 months follow up ( $(=1.15, p=.03)$. No statistically significant associations between spouses' report of providing higher negative dyadic coping at baseline and patients' relationship quality and 
depression was observed. This suggests that although patients' relationship quality was not observed to be affected by spouses' higher negative dyadic coping, they were more likely to experience at least one psychological distress outcome (refer Table 2B).

\section{Findings from Dyadic Growth Curve Model}

The findings suggested that patients who reported higher supportive dyadic coping, showed that on average patient's stress was 18.16 on a scale of 0 to 63 ; depression was 16.47 on a scale of 0 to 63 and relationship quality was 79.78 on a scale of 0 to 100 . One unit increase in supportive dyadic coping reduced patients' stress by -0.08 , depression by -0.20 and increased relationship quality by 1.82 . Similarly, findings for partners who reported higher supportive dyadic coping, showed that on average partner's stress was 9.33 on a scale of 0 to 63 ; depression was 13.44 on a scale of 0 to 63 and relationship quality was 91.44 on a scale of 0 to 100. One unit increase in supportive dyadic coping reduced partners' stress by -0.03 , depression by -0.31 and increased relationship quality by 1.30 (refer Table 3 ). Due to lack of convergence of the likelihood function the coefficient estimates for anxiety and supportive dyadic coping could not be computed.

Similarly in case of negative dyadic coping findings suggest that patients who reported higher negative dyadic coping, their average stress was 11.26 on a scale of 0 to 63 and depression was 7.45 on a scale of 0 to 63 . One unit increase in negative dyadic coping increased patients' stress by 0.77 and depression by 0.79 . Also, the findings for partners who reported higher negative dyadic coping, demonstrated that on average partner's stress was 7.15 on a scale of 0 to 63 and depression was 4.18 on a scale of 0 to 63 . One unit increase in negative dyadic coping increased partners' stress by 0.31 and depression by 0.56 (refer Table 3). Due to instability of estimates model for anxiety, relationship quality and negative dyadic coping for both patient and partner are not reported. 


\section{Discussion}

To the best of our knowledge, this is one of the first studies to examine the interpersonal process of dyadic coping (i.e., a process of coping that transpires between couples) from both the patients' and their spouses' perspective in the context of chronic pain. Using an innovative statistical model, the Actor-Partner Interdependence Model (APIM), the role of patientperceived and spouse-reported dyadic coping was examined in predicting patients' and spouses' relationship quality, anxiety, stress and depression over time.

The results showed that patients' perception of dyadic coping at baseline showed strong associations with their own, as well as their spouses' relationship quality, over the follow up period of 6 months. Especially patients' perception of receiving negative dyadic coping was inversely proportional to both patient and their spouses' relationship quality. This suggests that if patients perceived higher negative dyadic coping from their spouse at baseline (e.g., their spouse does not take their pain seriously, withdraws when the patient has pain, and/or blames the patient that s/he is not coping well with pain) it lowered both partners' relationship quality for up to 6 months. Likewise, patients' perception of receiving more negative coping efforts from their spouse showed, over time, adverse effects on their own report of depression and their spouses' report of depression at baseline and stress at 6 months follow up. In addition, spouses of patients who perceived receiving higher supportive dyadic coping (e.g., who perceived more help, sympathy and understanding from their spouse) reported lower stress over time.

Findings from this study also showed that spouses' own report of providing higher supportive dyadic coping was associated with their own relationship quality and depression at baseline but this association was lost over time. No immediate or over time effect of spouse-reported supportive dyadic coping was observed on patients' relationship quality or psychological outcomes, except for the report of depression at 3 months follow up. Also, spouse-reported 
negative dyadic coping was only associated with spouses' relationship quality and depression at baseline and anxiety and stress at 3 months follow up and patients' anxiety and stress at 6 months follow up.

Although some prior studies [38-40] have examined the association between dyadic coping and relationship quality, most studies have only focused on patients' report of relationship quality [41]. This is the first study to examine the transactional effects of dyadic coping, as indexed by patient-perceived and spouse-reported dyadic coping, on relationship quality of both patient and their spouse. In comparison to spouses' own report of providing dyadic coping, patients' perception of received dyadic coping seems to be a better predictor of relationship quality and psychological outcomes, for both patient and their spouse, over time.

Of note, in comparison to supportive dyadic coping, both perceived and reported negative dyadic coping had stronger associations with both patients' and their spouses' relationship quality and psychological distress. While previous studies have demonstrated that negative spousal support is related to affective distress and depression in chronic pain patients $[17,42]$, its longitudinal impact over time on both patient and their spouses was not known. The present findings suggest that negative dyadic coping, in comparison to supportive dyadic coping, exhibits stronger over time effects on relationship quality and psychological outcomes of couples coping with chronic pain. After accounting, for both temporal and interpersonal correlations using the dyadic growth curve model, we found that the inference remained similar as observed in the APIM model. To elaborate, report of higher supportive dyadic coping seems to lower depression and stress, while increasing relationship quality, for both members of the dyad.

Given the more modest correlations between supportive dyadic coping and outcomes, an interesting hypothesis that could be explored in further research is that supportive dyadic coping, as social resource for patients with chronic pain, may rather have a buffering effect 
instead of a direct effect on individual psychological outcomes [5, 43]. Some studies have observed that higher levels of social support magnify the negative effects of catastrophizing [44] and disability [45] on pain. However, in another study Ginting et al. [46] observed that social support buffered the negative effect of stress on all pain-related outcomes, especially mental health. This raises the possibility that, depending on the type of coping, pain-related social support may either buffer or amplify the detrimental effect of stress on pain experiences. This is yet to be explored and warrants further investigation.

The findings of the current study may have clinical implications. Firstly, the study findings show that spouses of chronic pain patients also reported significant psychological distress over time, which suggests that spouses may also benefit from early psychosocial intervention. Secondly, appropriate appraisal of partners' expectations from dyadic coping is critical to both patients' and their partners' overall wellbeing. Incorporating interventions to teach adaptive and open communication skills training to the couples may directly improve their individual psychological outcomes and indirectly their relationship quality. Lastly, it is important to recognize that the construct of dyadic coping between individuals who share an intimate relationship is not fully altruistic in nature but is intended to protect their relationship from any potential harms of the illness [10]. Therefore, focusing on strengthening the couple's relationship and bond may be an important target point for clinical practice as it shows close relationships with how couples cope, adapt and support each other when one partner suffers from chronic pain.

\section{Limitations and Future Directions}

We acknowledge several limitations of this study. Firstly, couples were recruited via self-help organizations and may not be representative of the general patient population seen in clinical settings. Second, most of the patients were females in a heterosexual relationship, which limits the generalizability of these results to patients of diverse communities. More work is needed to 
clarify the role of contextual factors such as sex, gender, and sexual orientation in dyadic coping and couple outcomes.

\section{Conclusions}

The present study findings suggest that patients' perception of dyadic coping in comparison to spouses' report is a better predictor of relationship quality and psychological outcomes, for both patient and their spouse, over time. These findings highlight the importance of early psychosocial intervention, which may help couples better appraise each other's expectations from dyadic coping, indirectly supporting better relationship quality and psychological outcomes for both patient and their spouse.

\section{Acknowledgements}

We would like to thank Flemish League for Fibromyalgia patients and Flemish pain league for their assistance with recruiting participants. This research was supported by the Fund for Scientific Research-Flanders (FWO), grant number G.0235.13N. MMM was funded by the 2018 Australian Government funded Endeavour Post-doctoral Research Fellowship. MNM was funded by the 2018 Australian Government funded Endeavour Executive Fellowship.

\section{Conflict of Interest}

The authors have no conflicts of interest to disclose.

\section{References}

1 Lutz J, Gross RT, Vargovich AM. Difficulties in emotion regulation and chronic painrelated disability and opioid misuse. Addict Behav 2018;87:200-5.

2 Outcalt SD, Kroenke K, Krebs EE, Chumbler NR, Wu J, Yu Z, Bair MJ. Chronic pain and comorbid mental health conditions: independent associations of posttraumatic stress disorder and depression with pain, disability, and quality of life. J Behav Med 2015;38(3):535-43. 
3 Lerman SF, Rudich Z, Brill S, Shalev H, Shahar G. Longitudinal associations between depression, anxiety, pain, and pain-related disability in chronic pain patients. Psychosom Med 2015;77(3):333-41.

4 Fayaz A, Croft P, Langford RM, Donaldson LJ, Jones GT. Prevalence of chronic pain in the UK: a systematic review and meta-analysis of population studies. BMJ open 2016;6(6):e010364.

5 Bernardes SF, Forgeron P, Fournier K, Reszel J. Beyond solicitousness: a comprehensive review on informal pain-related social support. Pain 2017;158(11), 2066-2076.

6 Kindt S, Vansteenkiste M, Brenning K, Goubert L. The Effects of Partners' Helping Motivation on Chronic Pain Patients' Functioning Over Time. J Pain 2019;20(3), 348-357.

7 Jensen MP, Moore MR, Bockow TB, Ehde DM, Engel JM. Psychosocial factors and adjustment to chronic pain in persons with physical disabilities: A systematic review. Arch Phys Med Rehabil 2011;92:146-160.

8 Leonard MT, Cano A, Johanssen AB. Chronic pain in a couples' context: A review and integration of theoretical models and empirical evidence. J Pain 2006;7:377-390.

9 Newton-John TR. Solicitousness and chronic pain: A critical review. Pain Rev 2002; 9:727

10 Bodenmann G. A systemic-transactional conceptualization of stress and coping in couples. Swiss J Psycholo 1995.

11 Bodenmann G. Dyadic coping-a systematic-transactional view of stress and coping among couples: Theory and empirical findings. Eur Rev Appl Psychol 1997;47:137-40.

12 Bodenmann G. Dyadic coping and its significance for marital functioning. Couples coping with stress: Emerging perspectives on dyadic coping 2005;1(1):33-50.

13 DeLongis A, Capreol M, Holtzman S, O'brien T, Campbell J. Social support and social strain among husbands and wives: a multilevel analysis. J Fam Psychol 2004;18(3):470. 
14 Zhang J, Yu NX, Zhou M, Zhang J. Dyadic effects of resilience on well-being in Chinese older couples: Mediating role of spousal support. J Fam Psychol 2017;31(3):273.

15 Huang J, Zhang J, Yu NX. Close relationships, individual resilience resources, and wellbeing among people living with HIV/AIDS in rural China. AIDS Care 2019;1-9.

16 Ferreira VM, Sherman AM. The relationship of optimism, pain and social support to wellbeing in older adults with osteoarthritis. Aging Ment Health 2007;11(1):89-98.

17 Montoya P, Larbig W, Braun C, Preissl H, Birbaumer N. Influence of social support and emotional context on pain processing and magnetic brain responses in fibromyalgia. Arthritis Rheum 2004;50(12):4035-44.

18 Revenson TA, Schiaffino KM, Majerovitz SD, Gibofsky A. Social support as a doubleedged sword: The relation of positive and problematic support to depression among rheumatoid arthritis patients. Soc Sci Med 1991;33(7):807-13.

19 Riemsma RP, Taal E, Rasker JJ. Perceptions about perceived functional disabilities and pain of people with rheumatoid arthritis: Differences between patients and their spouses and correlates with well-being. Arthritis Care Res 2000;13(5):255-61.

20 Cano A, Gillis M, Heinz W, Geisser M, Foran H. Marital functioning, chronic pain, and psychological distress. Pain 2004;107(1-2):99-106.

21 Katz J, Beach SR, Anderson P. Self-enhancement versus self-verification: Does spousal support always help?. Cognit Ther Res. 1996;20(4):345-60.

22 Uysal A, Ascigil E, Turunc G. Spousal autonomy support, need satisfaction, and well-being in individuals with chronic pain: A longitudinal study. J Behav Med 2017;40(2):281-92.

23 Bigatti SM, Cronan TA. An examination of the physical health, health care use, and psychological well-being of spouses of people with fibromyalgia syndrome. Health Psychol 2002;21(2):157. 
24 Prenevost $\mathrm{MH}$, Reme SE. Couples coping with chronic pain: How do intercouple interactions relate to pain coping?. Scand J Pain. 2017;16(1):150-7.

25 Von Korff M, Ormel J, Keefe FJ, Dworkin SF. Grading the severity of chronic pain. Pain 1992;50(2):133-49.

26 Gmelch S, Bodenmann G, Meuwly N, Ledermann T, Steffen-Sozinova O, Striegl K. Dyadic Coping Inventory (DCI): A questionnaire assessing dyadic coping in couples. Z Familienforsch 2008; 20:185-202.

27 Randall AK, Hilpert P, Jimenez-Arista LE, Walsh KJ, Bodenmann G. Dyadic coping in the US: Psychometric properties and validity for use of the English version of the Dyadic Coping Inventory. Curr Psychol 2016;35(4):570-82.

28 Levesque C, Lafontaine MF, Caron A, Fitzpatrick J. Validation of the English version of the Dyadic Coping Inventory. Measurement and Evaluation in Counseling and Development. 2014;47(3):215-25.

29 Rottmann N, Hansen DG, Larsen PV, Nicolaisen A, Flyger H, Johansen C, Hagedoorn M. Dyadic coping within couples dealing with breast cancer: A longitudinal, population-based study. Health Psychol. 2015;34(5):486.

30 de Beurs E, Van Dyck R, Marquenie LA, Lange A, Blonk RW. De DASS: een vragenlijst voor het meten van depressie, angst en stress. Gedragstherapie. 2001;34(1):35-54.

31 Lovibond PF, Lovibond SH. The structure of negative emotional states: Comparison of the Depression Anxiety Stress Scales (DASS) with the Beck Depression and Anxiety Inventories. Behav Res Therapy 1995;33(3):335-43.

32 Spanier GB. Measuring dyadic adjustment: New scales for assessing the quality of marriage and similar dyads. JMF 1976;15-28. 
33 Heene E, Buysse A, Van Oost P. Assessment van relationeel functioneren: de ontwikkeling van Nederlandstalig instrumentarium. Nederlands Tijdschrift Voor de Psychologie 2000;55, 203-216.

34 Leonard MT, Issner JH, Cano A, Williams AM. Correlates of spousal empathic accuracy for pain-related thoughts and feelings. Clin J Pain 2013;29(4):324-33.

35 Cook WL, Kenny DA. The actor-partner interdependence model: A model of bidirectional effects in developmental studies. Int J Behav Dev 2005;29(2):101-9.

36 Gonzalez R. A statistical framework for modeling homogeneity and interdependence in groups. Blackwell handbook of social psychology: Interpersonal processes. 2003;505-34.

37 Kenny DA, Kashy DA. Dyadic data analysis using multilevel modeling. In: Hox JJ, Roberts JK, eds. Handbook for advanced multilevel analysis. Routledge/Taylor \& Francis Group.2011; 335-370.

38 Rosen NO, Bergeron S, Sadikaj G, Glowacka M, Delisle I, Baxter ML. Impact of male partner responses on sexual function in women with vulvodynia and their partners: A dyadic daily experience study. Health Psychol 2014;33(8):823.

39 Revenson TA, DeLongis A. Couples coping with chronic illness. The Oxford handbook of stress, health, and coping 2011;101-23.

40 Traa MJ, De Vries J, Bodenmann G, Den Oudsten BL. Dyadic coping and relationship functioning in couples coping with cancer: a systematic review. $\mathrm{Br}$ J Health Psychol 2015;20(1):85-114.

41 Segrin C, Badger TA, Meek P, Lopez AM, Bonham E, Sieger A. Dyadic interdependence on affect and quality-of-life trajectories among women with breast cancer and their partners. J Soc Pers Relat 2005;22(5):673-89. 
42 Cano A, Weisberg JN, Gallagher RM. Marital satisfaction and pain severity mediate the association between negative spouse responses to pain and depressive symptoms in a chronic pain patient sample. Pain Med 2000;1(1):35-43.

43 Brandão T, Schulz MS, Matos PM. Psychological adjustment after breast cancer: a systematic review of longitudinal studies. Psycho-oncology 2017;26(7):917-26.

44 Giardino_2003 Giardino ND, Jensen MP, Turner JA, Ehde DM, Cardenas DD. Social environment moderates the association between catastrophizing and pain among persons with a spinal cord injury. Pain 2003;106:19-25.

45 Ginting JV, Tripp DA, Nickel CJ. Self-reported spousal support modifies the negative impact of pain on disability in men with chronic prostatitis/chronic pelvic pain syndrome. Urology 2011;78(5):1136-41.

46 Ginting JV, Tripp DA, Nickel JC, Fitzgerald MP, Mayer R. Spousal support decreases the negative impact of pain on mental quality of life in women with interstitial cystitis/painful bladder syndrome. BJU Int 2010;108:713-7. 
Table 1: Patient perceived dyadic coping and its effect on their own and their spouse's relationship quality and psychological distress

All analyses controlled for sex, relationship duration and pain intensity. *p value $<.05$, ** $p$ value $<.01$

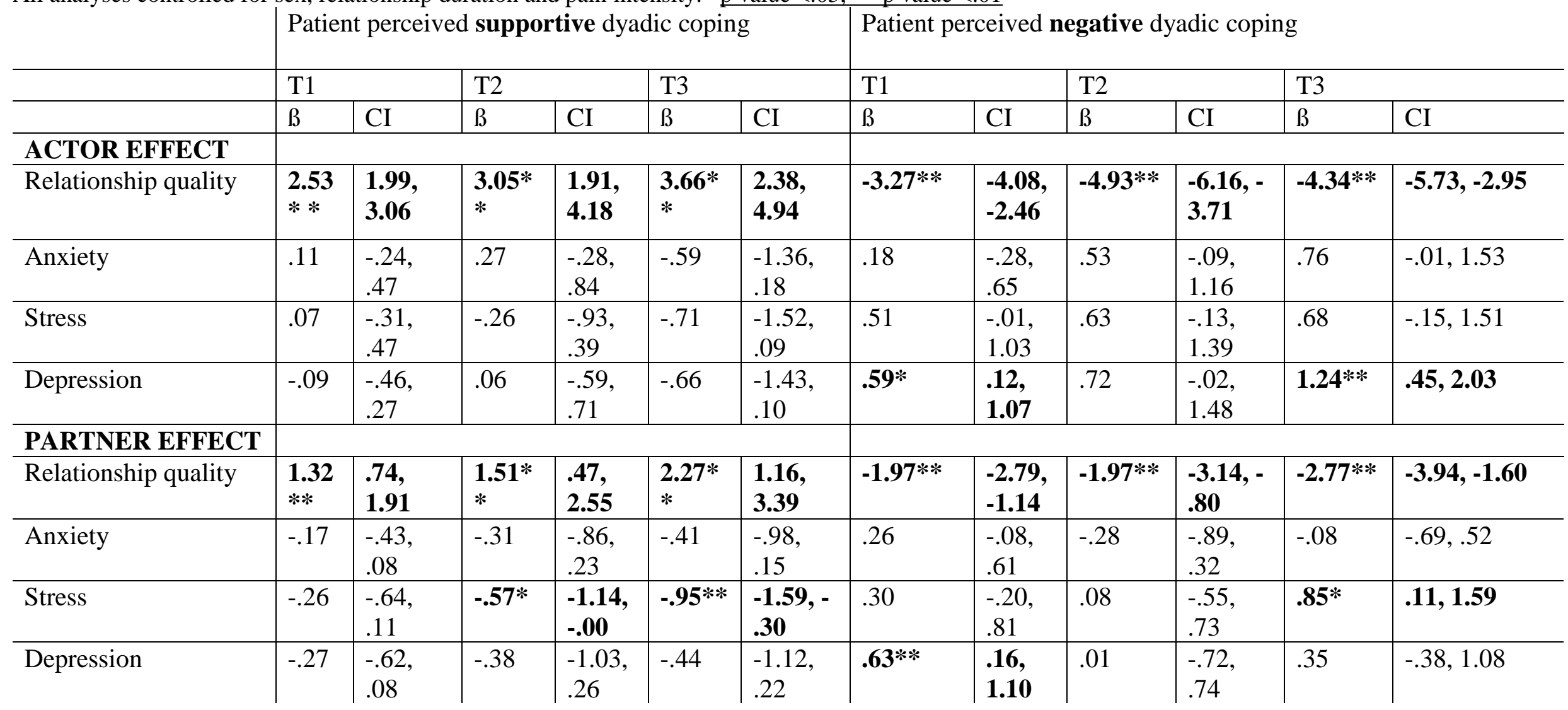


Table 2: Spouse-reported dyadic coping and its effect on their own and patient's relationship quality and psychological distress

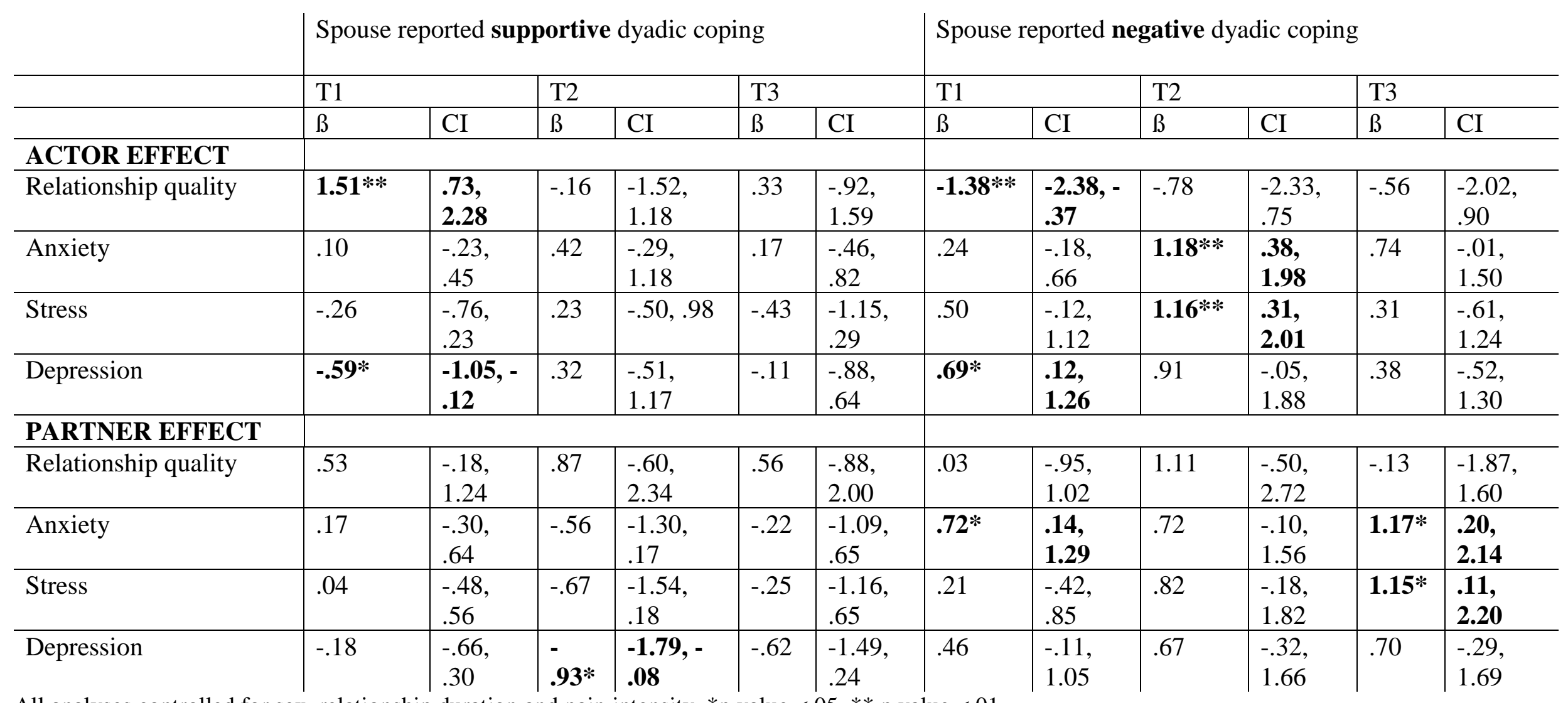

All analyses controlled for sex, relationship duration and pain intensity. *p value $<.05, * * p$ value $<.01$ 
Table 3: Findings from the Dyadic Growth Curve Model

\begin{tabular}{|c|c|c|c|c|c|c|c|}
\hline & & \multicolumn{2}{|l|}{ Stress } & \multicolumn{2}{|c|}{ Depression } & \multicolumn{2}{|c|}{ Relationship Quality } \\
\hline & & $\beta$ (coeff.) & SE & $\beta$ (coeff.) & SE & $\beta$ (coeff.) & SE \\
\hline \multirow[t]{2}{*}{ Supportive DC } & Patient & 18.16 & $2.36^{* *}$ & 16.47 & $2.30^{* *}$ & 79.78 & $3.26^{* *}$ \\
\hline & Partner & 9.33 & $2.70^{* *}$ & 13.44 & $2.54^{* *}$ & 91.44 & $3.99^{* *}$ \\
\hline \multirow[t]{2}{*}{ Negative DC } & Patient & 11.26 & $1.48^{* *}$ & 7.45 & $1.43^{* *}$ & - & \\
\hline & Partner & 7.15 & $1.46^{* *}$ & 4.18 & $1.36^{* *}$ & - & \\
\hline
\end{tabular}

${ }^{*} \mathrm{p}$ value $<.05, * * \mathrm{p}$ value $<.01$ 
Figure 1: Actor-Partner Interdependence M

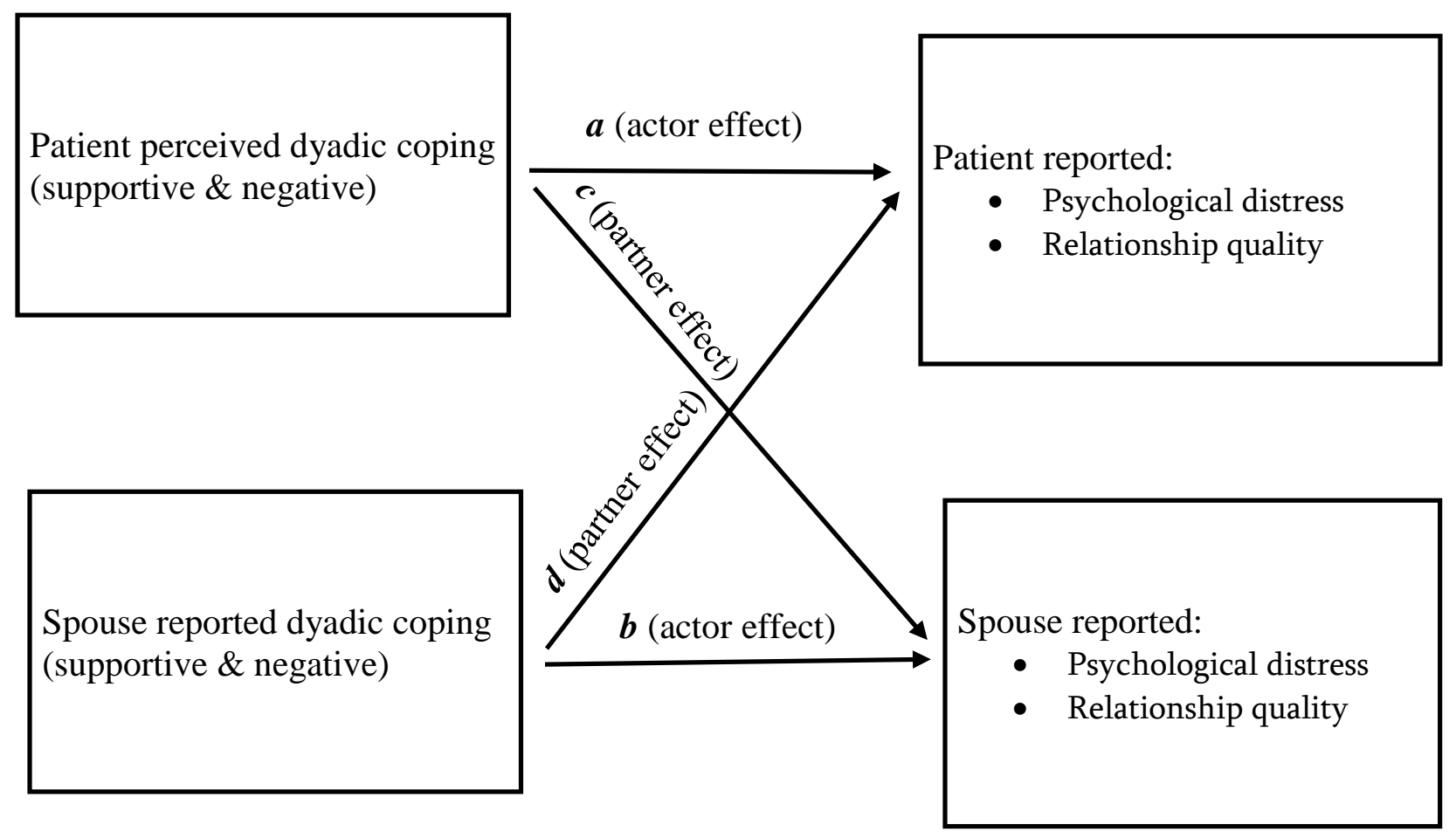


